\title{
SLOWLY COUPLED OSCILLATORS: PHASE DYNAMICS AND SYNCHRONIZATION*
}

\author{
EUGENE M. IZHIKEVICH ${ }^{\dagger}$ AND FRANK C. HOPPENSTEADT ${ }^{\ddagger}$
}

\begin{abstract}
In this paper we extend the results of Frankel and Kiemel [SIAM J. Appl. Math, 53 (1993), pp. 1436-1446] to a network of slowly coupled oscillators. First, we use Malkin's theorem to derive a canonical phase model that describes synchronization properties of a slowly coupled network. Then, we illustrate the result using slowly coupled oscillators (1) near Andronov-Hopf bifurcations, (2) near saddle-node on invariant circle bifurcations, and (3) near relaxation oscillations. We compare and contrast synchronization properties of slowly and weakly coupled oscillators.
\end{abstract}

Key words. phase model, Andronov-Hopf, saddle-node on invariant circle, Class 1 excitability, relaxation oscillators, Malkin theorem, MATLAB

AMS subject classifications. 92B20, 92C20, 82C 32 , 58Fxx, 34Cxx, 34Dxx

DOI. $10.1137 /$ S0036139902400945

1. Slowly coupled networks. In this paper we study synchronization dynamics of a network of $n \geq 2$ coupled oscillators of the form

$$
\begin{aligned}
\dot{x}_{i} & =f_{i}\left(x_{i}, s_{1}, \ldots, s_{n}\right), \\
\dot{s}_{i} & =\varepsilon g_{i}\left(x_{i}, s_{i}\right),
\end{aligned}
$$

where $x_{i} \in \mathbb{R}^{m}$ describes the state of the $i$ th oscillator and $s_{i} \in \mathbb{R}$ describes how the $i$ th oscillator affects the other oscillators for $i=1, \ldots, n$. The parameter $\varepsilon \ll 1$ is small reflecting the assumption that the connection variables $s_{i}$ are "slow." We analyze this system in this section and present several examples in section 2.

Proceeding as in Frankel and Kiemel (1993) we "freeze" the vector of slow variables $s=\left(s_{1}, \ldots, s_{n}\right) \in \mathbb{R}^{n}$ and assume that each oscillator described by (1.1) has a $T_{i}(s)$-periodic solution $x_{i}=x_{i}(t, s)$. Substituting this in (1.2) results in the system

$$
\dot{s}_{i}=\varepsilon g_{i}\left(x_{i}(t, s), s_{i}\right), \quad i=1, \ldots, n,
$$

which we can average and obtain a slow system

$$
\dot{s}_{i}=\varepsilon \bar{g}_{i}(s), \quad i=1, \ldots, n,
$$

where

$$
\bar{g}_{i}(s)=\frac{1}{T_{i}(s)} \int_{0}^{T_{i}(s)} g_{i}\left(x_{i}(t, s), s_{i}\right) d t
$$

is the average of $g_{i}$. In this paper we make the following two assumptions:

* Received by the editors January 14, 2002; accepted for publication (in revised form) February 28, 2003; published electronically September 4, 2003. This research was supported in part by NSF grant DMS-0109001.

http://www.siam.org/journals/siap/63-6/40094.html

${ }^{\dagger}$ The Neurosciences Institute, 10640 John Jay Hopkins Drive, San Diego, CA 92121 (Eugene. Izhikevich@nsi.edu, http://www.izhikevich.com). The research of this author was carried out as part of the theoretical neurobiology program at The Neurosciences Institute, which is supported by the Neurosciences Research Foundation.

${ }^{\ddagger}$ Systems Science and Engineering Research Center, Arizona State University, Tempe, AZ 852877606 (fchoppen@asu.edu.). 
A1. The system (1.3) has an exponentially stable equilibrium $\bar{s}=\left(\bar{s}_{1}, \ldots, \bar{s}_{n}\right)$.

A2. Each equation (1.1) has an exponentially stable limit cycle attractor $\gamma_{i}(t) \subset$ $\mathbb{R}^{m}$ with period $T>0$ when $s=\bar{s}$.

TheOREM 1.1 (phase model for slowly coupled oscillators). Consider the slowly coupled system (1.1), (1.2) satisfying assumptions A1 and A2 above. Let $\tau=\varepsilon t$ be slow time. Let $u_{i}(\tau)$ be the rescaled deviation of the slow variable $s_{i}$ from the asymptotic value $\bar{s}_{i}$, and let $\varphi_{i}(\tau)$ be the phase deviation of the ith oscillator from the natural oscillation $\gamma_{i}(t)$. Then, the phase dynamics and synchronization properties of the slowly coupled system (1.1), (1.2) are described by the canonical phase model

$$
\begin{aligned}
\varphi_{i}^{\prime} & =\sum_{j=1}^{n}\left\{a_{i j} u_{j}+H_{i j}\left(\varphi_{j}-\varphi_{i}\right)\right\}, \\
u_{i}^{\prime} & =\sum_{j=1}^{n}\left\{b_{i j} u_{j}+K_{i j}\left(\varphi_{j}-\varphi_{i}\right)\right\},
\end{aligned}
$$

where ${ }^{\prime}=d / d \tau$, and

$$
\begin{aligned}
a_{i j} & =\frac{1}{T} \int_{0}^{T} Q_{i}(t)^{\top} \frac{\partial f_{i}}{\partial s_{j}}\left(\gamma_{i}(t), \bar{s}\right) d t \\
b_{i j} & =\frac{1}{T} \int_{0}^{T}\left[P_{i}(t)^{\top} \frac{\partial f_{i}}{\partial s_{j}}\left(\gamma_{i}(t), \bar{s}\right)+\frac{\partial g_{i}}{\partial s_{j}}\left(\gamma_{i}(t), \bar{s}_{i}\right)\right] d t \\
H_{i j}(\chi) & =\frac{1}{T} \int_{0}^{T} Q_{i}(t)^{\top} \frac{\partial f_{i}}{\partial s_{j}}\left(\gamma_{i}(t), \bar{s}\right) \int_{0}^{t+\chi} g_{j}\left(\gamma_{j}(\bar{t}), \bar{s}\right) d \bar{t} d t, \\
K_{i j}(\chi) & =\frac{1}{T} \int_{0}^{T}\left(P_{i}(t)^{\top} \frac{\partial f_{i}}{\partial s_{j}}\left(\gamma_{i}(t), \bar{s}\right)+\frac{\partial g_{i}}{\partial s_{j}}\left(\gamma_{i}(t), \bar{s}_{i}\right)\right) \int_{0}^{t+\chi} g_{j}\left(\gamma_{j}(\bar{t}), \bar{s}\right) d \bar{t} d t,
\end{aligned}
$$

where $Q_{i}(t), P_{i}(t) \subset \mathbb{R}^{m}$ are the unique nontrivial T-periodic solutions of the linear adjoint systems

$$
\dot{Q}_{i}=-\left\{\frac{\partial f_{i}}{\partial x_{i}}\left(\gamma_{i}(t), \bar{s}\right)\right\}^{\top} Q_{i} \quad \text { and } \quad \dot{P}_{i}=-\left\{\frac{\partial f_{i}}{\partial x_{i}}\left(\gamma_{i}(t), \bar{s}\right)\right\}^{\top} P_{i}-\left\{\frac{\partial g_{i}}{\partial x_{i}}\left(\gamma_{i}(t), \bar{s}\right)\right\}^{\top}
$$

satisfying the normalization conditions

$$
Q_{i}(t)^{\top} f_{i}\left(\gamma_{i}(t), \bar{s}\right)=1 \quad \text { and } \quad P_{i}(t)^{\top} f_{i}\left(\gamma_{i}(t), \bar{s}\right)=-g_{i}\left(\gamma_{i}(t), \bar{s}\right)
$$

for some (and hence all) $t \geq 0$.

Remark 1.2. The same result holds when $g_{i}\left(x_{i}, s_{i}\right)$ also depend on $s_{1}, \ldots, s_{n}$.

Remark 1.3. The same result holds for the slowly and weakly coupled system

$$
\begin{aligned}
& \dot{x}_{i}=f_{i}\left(x_{i}, s_{1}, \ldots, s_{n}\right)+\varepsilon \sum_{j=1}^{n} r_{i j}\left(x_{i}, x_{j}\right), \\
& \dot{s}_{i}=\varepsilon g_{i}\left(x_{i}, s_{i}\right),
\end{aligned}
$$

provided that the term

$$
\frac{1}{T} \int_{0}^{T} Q_{i}(t)^{\top} r_{i j}\left(\gamma_{i}(t), \gamma_{j}(t+\chi)\right) d t
$$


is added to the function $H_{i j}(\chi)$ and the term

$$
\frac{1}{T} \int_{0}^{T} P_{i}(t)^{\top} r_{i j}\left(\gamma_{i}(t), \gamma_{j}(t+\chi)\right) d t
$$

is added to the function $K_{i j}(\chi)$.

Remark 1.4. This result not only extends the result of Frankel and Kiemel (1993) to a network of $n \geq 2$ oscillators, but also presents a precise description of all the parameters and functions in the canonical model (1.4), (1.5), which can easily be determined numerically; see Appendix B.

Proof. This result is a corollary to Malkin's theorem, which we restate in Appendix A. Consider the slowly coupled system (1.1), (1.2) in an $\varepsilon$-neighborhood of $\bar{s}$. Let

$$
s_{i}=\bar{s}_{i}+\varepsilon w_{i},
$$

so that we can rewrite (1.1), (1.2) in the form (A.1),

$$
\begin{aligned}
& \dot{x}_{i}=f_{i}\left(x_{i}, \bar{s}\right)+\varepsilon \sum_{j=1}^{n} h_{i j}\left(x_{i}, \bar{s}\right) w_{j}, \\
& \dot{w}_{i}=g_{i}\left(x_{i}, \bar{s}_{i}\right)+\varepsilon p_{i}\left(x_{i}, \bar{s}_{i}\right) w_{i}
\end{aligned}
$$

plus higher-order terms in $\varepsilon$, where

$$
h_{i j}=\frac{\partial f_{i}}{\partial s_{j}} \quad \text { and } \quad p_{i}=\frac{\partial g_{i}}{\partial s_{i}} .
$$

In the rest of the proof we omit $\bar{s}$ for the sake of clarity of notation.

Since (1.8), (1.9) has a "weakly connected" form, it suffices to show that all the conditions of Malkin's theorem are satisfied for each individual oscillator.

Each unperturbed (uncoupled, $\varepsilon=0$ ) system

$$
\begin{aligned}
\dot{x}_{i} & =f_{i}\left(x_{i}\right), \\
\dot{w}_{i} & =g_{i}\left(x_{i}\right)
\end{aligned}
$$

has a 2-parameter family of $T$-periodic solutions

$$
x_{i}(t)=\gamma_{i}\left(t+\varphi_{i}\right) \quad \text { and } \quad w_{i}(t)=u_{i}+\int_{0}^{t+\varphi_{i}} g_{i}\left(\gamma_{i}(\bar{t})\right) d \bar{t}
$$

where $\varphi_{i}$ and $u_{i}$ are independent parameters, $i=1, \ldots, n$. $\left(w_{i}(t)\right.$ is periodic because $s_{i}$ is at equilibrium and the average of $g_{i}$ over the limit cycle is assumed to be zero.) Let $Q_{i}(t)$ and $P_{i}(t)$ be the unique nontrivial solutions to the adjoint system (1.6), which exist because $\gamma_{i}(t)$ is a normally hyperbolic attractor (Hoppensteadt and Izhikevich 1997). One can verify that

$$
R_{i}(t)=\left(\begin{array}{c}
Q_{i}(t) \\
0
\end{array}\right) \quad \text { and } \quad R_{i}(t)=\left(\begin{array}{c}
P_{i}(t) \\
1
\end{array}\right)
$$

are two independent nontrivial solutions to the adjoint system (A.2),

$$
\dot{R}_{i}=-\left(\begin{array}{cc}
\frac{\partial f_{i}}{\partial x_{i}}\left(\gamma_{i}(t)\right) & 0 \\
\frac{\partial g_{i}}{\partial x_{i}}\left(\gamma_{i}(t)\right) & 0
\end{array}\right)^{\top} R_{i}
$$


Equation (A.4) results in

$$
\begin{aligned}
\varphi_{i}^{\prime} & =\frac{1}{T} \int_{0}^{T} Q_{i}\left(t+\varphi_{i}\right)^{\top}\left\{\sum_{j=1}^{n} h_{i j}\left(\gamma_{i}\left(t+\varphi_{i}\right)\right)\left(u_{j}+\int_{0}^{t+\varphi_{j}} g_{j}\left(\gamma_{j}(\bar{t})\right) d \bar{t}\right)\right\} d t \\
& =\frac{1}{T} \int_{0}^{T} Q_{i}(t)^{\top}\left\{\sum_{j=1}^{n} h_{i j}\left(\gamma_{i}(t)\right)\left(u_{j}+\int_{0}^{t+\varphi_{j}-\varphi_{i}} g_{j}\left(\gamma_{j}(\bar{t})\right) d \bar{t}\right)\right\} d t
\end{aligned}
$$

and

$$
\begin{gathered}
u_{i}^{\prime}=\frac{1}{T} \int_{0}^{T} P_{i}\left(t+\varphi_{i}\right)^{\top}\left\{\sum_{j=1}^{n} h_{i j}\left(\gamma_{i}\left(t+\varphi_{i}\right)\right)\left(u_{j}+\int_{0}^{t+\varphi_{j}} g_{j}\left(\gamma_{j}(\bar{t})\right) d \bar{t}\right)\right\} \\
+1 \cdot\left\{p_{i}\left(\gamma_{i}\left(t+\varphi_{i}\right)\right)\left(u_{i}+\int_{0}^{t+\varphi_{i}} g_{i}\left(\gamma_{i}(\bar{t})\right) d \bar{t}\right)\right\} d t \\
=\frac{1}{T} \int_{0}^{T} P_{i}(t)^{\top}\left\{\sum_{j=1}^{n} h_{i j}\left(\gamma_{i}(t)\right)\left(u_{j}+\int_{0}^{t+\varphi_{j}-\varphi_{i}} g_{j}\left(\gamma_{j}(\bar{t})\right) d \bar{t}\right)\right\} \\
+1 \cdot\left\{p_{i}\left(\gamma_{i}(t)\right)\left(u_{i}+\int_{0}^{t+\varphi_{j}-\varphi_{i}} g_{i}\left(\gamma_{i}(\bar{t})\right) d \bar{t}\right)\right\} d t,
\end{gathered}
$$

which can be written in the form (1.4), (1.5).

2. Examples. The major challenge in applying Theorem 1.1 is solving the linear adjoint system (1.6). In general, this could be done numerically, as we show in Appendix B. However, there are three important cases when (1.6) can be solved analytically:

- Each oscillator is near an Andronov-Hopf bifurcation.

- Each oscillator is near a saddle-node on invariant circle bifurcation.

- Each oscillator has two time scales (relaxation oscillator).

We consider all three cases below, but first we start with two simple examples.

2.1. Phase oscillators. The system of slowly coupled phase oscillators

$$
\begin{aligned}
& \dot{\vartheta}_{i}=1+\sum_{j=1}^{n} c_{i j} s_{j}, \\
& \dot{s}_{i}=\varepsilon\left(\cos \vartheta_{i}-b s_{i}\right)
\end{aligned}
$$

illustrates the major steps in Theorem 1.1. When all $s_{i}$ are not very large, the averaged slow system (1.3) has the form

$$
\dot{s}_{i}=-\varepsilon b s_{i} .
$$

If $b>0, \bar{s}=0 \in \mathbb{R}^{n}$ is an exponentially stable equilibrium, and assumption A1 is satisfied. Assumption A2 is also satisfied, since at $\bar{s}=0$ all phase oscillators, described by $\dot{\vartheta}_{i}=1$, have equal period $T=2 \pi$. Let $s_{i}=\varepsilon w_{i}$; then system (1.8), (1.9) has the form

$$
\begin{aligned}
& \dot{\vartheta}_{i}=1+\varepsilon \sum_{j=1}^{n} c_{i j} w_{j}, \\
& \dot{w}_{i}=\cos \vartheta_{i}-\varepsilon b w_{i} .
\end{aligned}
$$


When $\varepsilon=0$, this system has a family of solutions

$$
\vartheta_{i}(t)=t+\varphi_{i} \quad \text { and } \quad w_{i}(t)=u_{i}+\sin \left(t+\varphi_{i}\right) .
$$

Since $\partial f_{i} / \partial \vartheta_{i}=0$, the adjoint system (1.6) has solutions $Q_{i}(t)=1$ and $P_{i}(t)=$ $-\cos t$, which satisfy the normalization condition (1.7). It is easy to check that

$$
\begin{aligned}
a_{i j} & =\frac{1}{T} \int_{0}^{T} 1 \cdot c_{i j} d t=c_{i j}, \\
b_{i j} & =\frac{1}{T} \int_{0}^{T}\left[-\cos t \cdot c_{i j}+0\right] d t=0, \quad i \neq j, \\
b_{i i} & =\frac{1}{T} \int_{0}^{T}\left[-\cos t \cdot c_{i i}-b\right] d t=-b, \\
H_{i j}(\chi) & =\frac{1}{T} \int_{0}^{T}\left[1 \cdot c_{i j} \cdot \int_{0}^{t+\chi} \cos \bar{t} d \bar{t}\right] d t=0, \\
K_{i j}(\chi) & =\frac{1}{T} \int_{0}^{T}\left[-\cos t \cdot c_{i j} \int_{0}^{t+\chi} \cos \bar{t} d \bar{t}\right] d t=-\frac{c_{i j}}{2} \sin \chi, \quad i \neq j,
\end{aligned}
$$

and $K_{i i}(0)=0$, so that the canonical phase model (1.4), (1.5) has the form

$$
\begin{aligned}
& \varphi_{i}^{\prime}=\sum_{j=1}^{n} c_{i j} u_{j}, \\
& u_{i}^{\prime}=-b u_{i}-\frac{1}{2} \sum_{j=1}^{n} c_{i j} \sin \left(\varphi_{j}-\varphi_{i}\right) .
\end{aligned}
$$

It is a simple exercise to check that the same canonical model can be obtained via standard averaging of (2.1), (2.2).

2.2. Frankel and Kiemel's example. As an illustration, Frankel and Kiemel (1993) considered the six-dimensional system

$$
\begin{aligned}
\dot{\vartheta}_{i} & =1+s_{j}\left(\alpha+\beta r_{i} \cos \vartheta_{i}+\gamma r_{i}^{2} \cos ^{2} \vartheta_{i}\right), \\
\dot{r}_{i} & =r_{i}-r_{i}^{3}+\eta s_{j} r_{i}^{2} \cos \vartheta_{i}, \\
\dot{s}_{i} & =\varepsilon\left(r_{i} \cos \vartheta_{i}-\mu s_{i}\right)
\end{aligned}
$$

having fast variables in polar coordinates $\mathbb{S}^{1} \times \mathbb{R}$ and parameters $\alpha, \beta, \gamma, \eta, \mu \in \mathbb{R}$, and $i, j \in\{1,2\}, i \neq j$. They used a completely different approach to show how the model can be reduced to the planar system

$$
\begin{aligned}
& \chi^{\prime}=(-\alpha-\gamma / 2) u-\beta \sin \chi, \\
& u^{\prime}=(\beta / 2-\eta / 5-\mu) u+(\alpha+3 \gamma / 4) \sin \chi,
\end{aligned}
$$

where

$$
\chi=\varphi_{2}-\varphi_{1} \quad \text { and } \quad u=u_{2}-u_{1}
$$

and $u_{i}$ and $\varphi_{i}$ have the same meaning as in this paper. 
Let us verify Frankel and Kiemel's result using Theorem 1.1. Each uncoupled oscillator has a $2 \pi$-periodic solution $(t, 1) \in \mathbb{S}^{1} \times \mathbb{R}$ when $\bar{s}=0$. It is easy to check by differentiating that the adjoint linear systems (1.6),

$$
\dot{Q}=-\left\{\begin{array}{cc}
0 & 0 \\
0 & -2
\end{array}\right\}^{\top} Q \quad \text { and } \quad \dot{P}=-\left\{\begin{array}{cc}
0 & 0 \\
0 & -2
\end{array}\right\}^{\top} P-\{-\sin t, \quad \cos t\}^{\top},
$$

have solutions

$$
Q(t)=\left(\begin{array}{l}
1 \\
0
\end{array}\right) \quad \text { and } \quad P(t)=\left(\begin{array}{c}
-\cos t \\
\frac{2}{5} \cos t-\frac{1}{5} \sin t
\end{array}\right)
$$

satisfying the normalization conditions (1.7). Therefore, the parameters in the canonical model (1.4), (1.5) are (here $i \neq j$ )

$$
\begin{aligned}
a_{i j} & =\frac{1}{2 \pi} \int_{0}^{2 \pi}\left(\begin{array}{c}
1 \\
0
\end{array}\right)^{\top}\left(\begin{array}{c}
\alpha+\beta \cos t+\gamma \cos ^{2} t \\
\eta \cos t
\end{array}\right) d t=\alpha+\frac{\gamma}{2}, \\
b_{i j} & =\frac{1}{2 \pi} \int_{0}^{2 \pi}\left(\begin{array}{c}
-\cos t \\
\frac{2}{5} \cos t-\frac{1}{5} \sin t
\end{array}\right)^{\top}\left(\begin{array}{c}
\alpha+\beta \cos t+\gamma \cos ^{2} t \\
\eta \cos t
\end{array}\right) d t=-\frac{\beta}{2}+\frac{\eta}{5}, \\
H_{i j}(\chi) & =\frac{1}{2 \pi} \int_{0}^{2 \pi}\left(\begin{array}{c}
1 \\
0
\end{array}\right)^{\top}\left(\begin{array}{c}
\alpha+\beta \cos t+\gamma \cos ^{2} t \\
\eta \cos t
\end{array}\right) \int_{0}^{t+\chi} \cos \bar{t} d \bar{t} d t=\frac{\beta}{2} \sin \chi, \\
K_{i j}(\chi) & =\frac{1}{2 \pi} \int_{0}^{2 \pi}\left(\begin{array}{c}
-\cos t \\
\frac{2}{5} \cos t-\frac{1}{5} \sin t
\end{array}\right)^{\top}\left(\begin{array}{c}
\alpha+\beta \cos t+\gamma \cos ^{2} t \\
\eta \cos t
\end{array}\right) \int_{0}^{t+\chi} \cos \bar{t} d \bar{t} d t \\
& =\left(-\frac{\alpha}{2}-\frac{3}{8} \gamma\right) \sin \chi,
\end{aligned}
$$

and

$$
a_{i i}=0, \quad b_{i i}=-\mu, \quad H_{i i}(0)=0, \quad \text { and } \quad K_{i i}(0)=-1 / 2 .
$$

Using the difference variables (2.5) we arrive exactly at the same model (2.3), (2.4) that Frankel and Kiemel did.

2.3. Andronov-Hopf bifurcation. Next, we derive the canonical phase model for a network of slowly coupled oscillators near an Andronov-Hopf bifurcation. Without loss of generality we may assume that $\bar{s}=0$ and that each oscillator has already been converted into the topological normal form (by a continuous near-identity change of variables; see Hoppensteadt and Izhikevich (1997)). We use complex coordinates for convenience and consider the system

$$
\begin{array}{ll}
\dot{z}_{i}=(\mu+\mathrm{i}) z_{i}-z_{i}\left|z_{i}\right|^{2}+q_{i}\left(z_{i}, \bar{z}_{i}, s_{1}, \ldots, s_{n}\right), & z_{i} \in \mathbb{C}, \\
\dot{s}_{i}=\varepsilon g_{i}\left(z_{i}, \bar{z}_{i}, s_{i}\right), & s_{i} \in \mathbb{R},
\end{array}
$$

where $\mathrm{i}=\sqrt{-1}$ has a different font from the subscript $i, 0<\varepsilon \ll \mu \ll 1$ (we assume $\mu$ is sufficiently small so that higher-order terms in $\mu$ may be neglected) and $q_{i}$ and $g_{i}$ are arbitrary smooth functions satisfying

$$
q_{i}\left(z_{i}, \bar{z}_{i}, 0, \ldots, 0\right)=0 \quad \text { and } \quad g_{i}(0,0,0)=0 .
$$

In this case each unperturbed $(\varepsilon=0)$ oscillator

$$
\dot{z}_{i}=(\mu+\mathrm{i}) z_{i}-z_{i}\left|z_{i}\right|^{2}
$$


has a small amplitude limit cycle attractor

$$
\gamma_{i}(t)=\sqrt{\mu} e^{i t} \subset \mathbb{C}
$$

with period $T=2 \pi$. We do not need to solve the adjoint linear system (1.6),

$$
\dot{Q}_{i}=-\{\mathrm{i}+\mathcal{O}(\mu)\}^{*} Q_{i} \quad \text { and } \quad \dot{P}_{i}=-\{\mathrm{i}+\mathcal{O}(\mu)\}^{*} P_{i}-\left\{\partial g_{i} / \partial z_{i}\right\}^{*},
$$

since we can find the solutions

$$
Q_{i}(t)=\mathrm{i} e^{\mathrm{i} t} / \sqrt{\mu} \quad \text { and } \quad P_{i}(t)=-\mathrm{i} e^{\mathrm{i} t}\left\{e^{\mathrm{i} t} \partial g_{i} / \partial z_{i}+\text { c.c. }\right\}
$$

directly from the normalization condition (1.7),

$$
Q_{i}(t)^{*}\left\{(\mu+\mathrm{i}) \gamma_{i}(t)-\gamma_{i}(t)\left|\gamma_{i}(t)\right|^{2}\right\}=Q_{i}(t)^{*}\left\{\mathrm{i} \sqrt{\mu} e^{\mathrm{i} t}\right\}=1
$$

and

$$
P_{i}(t)^{*}\left\{\mathrm{i} \sqrt{\mu} e^{\mathrm{i} t}\right\}=-g_{i}\left(\sqrt{\mu} e^{\mathrm{i} t}, 0\right)=-\left\{\sqrt{\mu} e^{\mathrm{i} t} \partial g_{i} / \partial z_{i}+\text { c.c. }\right\},
$$

where $Q_{i}(t), P_{i}(t) \in \mathbb{C},{ }^{*}$ denotes transposition and complex conjugation, and c.c. means complex-conjugate. Now we can apply Theorem 1.1 to obtain the canonical phase model

$$
\begin{aligned}
\varphi_{i}^{\prime} & =\sum_{j=1}^{n}\left\{a_{i j} u_{j}+c_{i j} \sin \left(\varphi_{j}+\psi_{i j}-\varphi_{i}\right)\right\} \\
u_{i}^{\prime} & =\sum_{j=1}^{n} b_{i j} u_{j}
\end{aligned}
$$

where

$$
\begin{aligned}
a_{i j} & =\operatorname{Im} \frac{\partial^{2} q_{i}}{\partial s_{j} \partial z_{i}}, \\
b_{i j} & =-\operatorname{Im} \frac{\partial q_{i}}{\partial s_{j}} \frac{\partial g_{i}}{\partial z_{i}}, \quad i \neq j \\
b_{i i} & =-\operatorname{Im} \frac{\partial q_{i}}{\partial s_{i}} \frac{\partial g_{i}}{\partial z_{i}}+\frac{\partial g_{i}}{\partial s_{i}},
\end{aligned}
$$

and all derivatives are evaluated at the origin $z=0$ and $s=0$. Notice that $P_{i}=\mathcal{O}(1)$ and $g_{i}=\mathcal{O}(\sqrt{\mu})$, hence $K_{i j}=\mathcal{O}(\sqrt{\mu})$ is infinitesimal. Let us show that

$$
H_{i j}(\chi)=c_{i j} \sin \left(\psi_{i j}+\chi\right) \text {. }
$$

First,

$$
\begin{aligned}
\int_{0}^{t+\chi} g_{j}\left(\sqrt{\mu} e^{\mathrm{i} \bar{t}}, \sqrt{\mu} e^{-\mathrm{i} \bar{t}}, 0\right) d \bar{t} & =\int_{0}^{t+\chi} \frac{\partial g_{j}}{\partial z_{j}} \sqrt{\mu} e^{\mathrm{i} \bar{t}} d \bar{t}+\text { c.c. } \\
& =\mathrm{i} \sqrt{\mu} \frac{\partial g_{j}}{\partial z_{j}}\left(1-e^{\mathrm{i}(t+\chi)}\right)+\text { c.c. }
\end{aligned}
$$




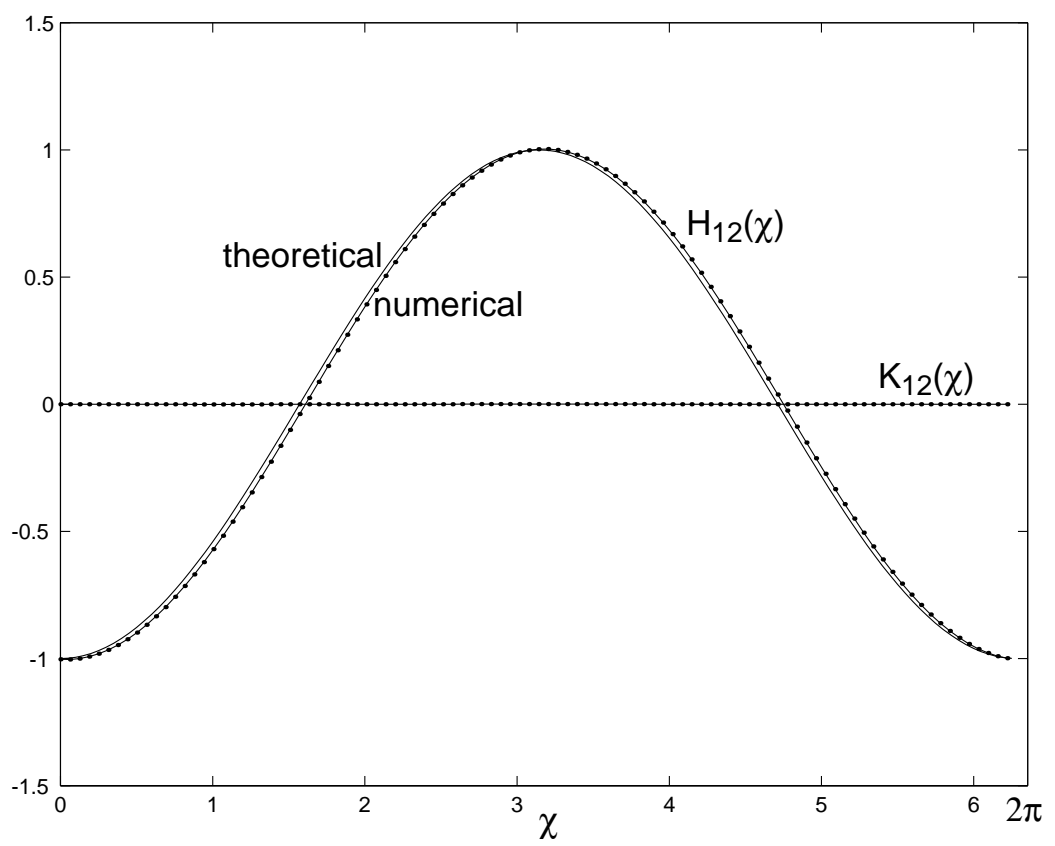

FIG. 2.1. Numerical (see Appendix B) and analytical form of the connection functions for slowly coupled oscillators near an Andronov-Hopf bifurcation. Parameters: $q_{1}\left(z_{1}, \bar{z}_{1}, s_{1}, s_{2}\right)=s_{2}$ and $g_{1}\left(z_{1}, \bar{z}_{1}, s_{1}\right)=z_{1}+\bar{z}_{1}-s_{1}, \mu=0.01$.

Next,

$$
\begin{aligned}
H_{i j}(\chi) & =\operatorname{Re} \frac{1}{2 \pi} \int_{0}^{2 \pi}\left(\frac{-\mathrm{i} e^{-\mathrm{i} t}}{\sqrt{\mu}}\right)\left(\frac{\partial q_{i}}{\partial s_{j}}+\mathcal{O}(\sqrt{\mu})\right)\left(\mathrm{i} \sqrt{\mu} \frac{\partial g_{j}}{\partial z_{j}}\left(1-e^{\mathrm{i}(t+\chi)}\right)+\text { c.c. }\right) d t \\
& =\operatorname{Re} \frac{1}{2 \pi} \int_{0}^{2 \pi}\left(-\frac{\partial q_{i}}{\partial s_{j}} \frac{\partial g_{j}}{\partial z_{j}} e^{\mathrm{i} \chi}+\text { terms involving } e^{\mathrm{i} t}\right) d t \\
& =-\operatorname{Re} \frac{\partial q_{i}}{\partial s_{j}} \frac{\partial g_{j}}{\partial z_{j}} e^{\mathrm{i} \chi}=c_{i j} \sin \left(\psi_{i j}+\chi\right),
\end{aligned}
$$

where

$$
c_{i j}=\left|\frac{\partial q_{i}}{\partial s_{j}} \frac{\partial g_{j}}{\partial z_{j}}\right| \quad \text { and } \quad \psi_{i j}=\operatorname{Arg} \frac{\partial q_{i}}{\partial s_{j}} \frac{\partial g_{j}}{\partial z_{j}}-\frac{\pi}{2}
$$

A typical example of the connection function $H_{i j}(\chi)$ is depicted in Figure 2.1. (Because theoretical and numerical curves were obtained using essentially the same formulae, this figure illustrates only the accuracy of the numerical method, and it does not validate the theory.)

Since the connection function $K_{i j}(\chi)=0$ for all $\chi$, the variables $u_{i}$ do not depend on the phase variables $\varphi_{i}$. If the matrix $\left(b_{i j}\right)$ is stable, all $u_{i}(t) \rightarrow 0$ as $t \rightarrow \infty$, and the locking properties of the slowly connected network are described by Kuramoto's (1984) system

$$
\varphi_{i}^{\prime}=\sum_{j=1}^{n} c_{i j} \sin \left(\varphi_{j}+\psi_{i j}-\varphi_{i}\right)
$$



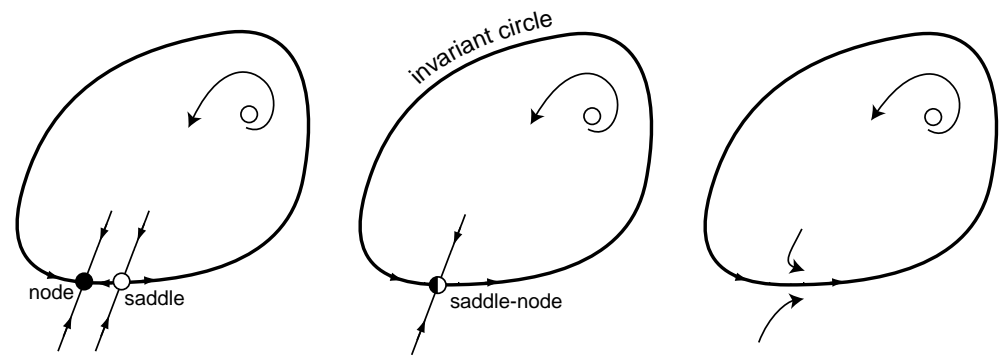

FIG. 2.2. Saddle-node on invariant circle bifurcation.

which was originally derived for weakly connected networks of Andronov-Hopf oscillators. Notice, though, that the variables $u_{i}$ may significantly slow down the convergence to a synchronized state if the matrix $\left(b_{i j}\right)$ has eigenvalues close to the imaginary axis.

2.4. Saddle-node on invariant circle bifurcation. Now we consider a slowly connected network of oscillators near a saddle-node on invariant circle bifurcation, which is illustrated in Figure 2.2. This bifurcation results in Class 1 excitable systems, i.e., systems able to oscillate with arbitrary small frequency (Hoppensteadt and Izhikevich (1997)). Without loss of generality we assume that $\bar{s}=0$ and that each oscillator has been restricted to the center manifold and converted to the topological normal form by an appropriate change of variables,

$$
\begin{array}{ll}
\dot{y}_{i}=\mu+y_{i}^{2}+q_{i}\left(y_{i}, c_{1}, \ldots, c_{n}, \mu\right), & y_{i} \in \mathbb{R}, \\
\dot{c}_{i}=\epsilon g_{i}\left(y_{i}, c_{i}\right), & c_{i} \in \mathbb{R},
\end{array}
$$

where $\epsilon \ll \sqrt{\mu} \ll 1$ and

$$
q_{i}\left(y_{i}, 0, \ldots, 0, \mu\right)=0 \quad \text { and } \quad g_{i}(0,0)=0 .
$$

Here we consider only a small neighborhood of the origin, since the spike lemma (Lemma 8.1 in Hoppensteadt and Izhikevich (1997)) implies that $y_{i}$ spends a negligible amount of time outside the small neighborhood; that is, action potentials generated by such a model look instantaneous on the slow time scale of order $1 / \sqrt{\mu}$. Now we rescale the variables and parameters,

$$
y_{i}=2 \sqrt{\mu} x_{i}, \quad c_{i}=2 \sqrt{\mu}\left\{\partial g_{i} / \partial y_{i}\right\} s_{i}, \quad \epsilon=2 \sqrt{\mu} \varepsilon, \quad t_{\text {new }}=2 \sqrt{\mu} t_{\text {old }},
$$

to transform the system above into the form

$$
\begin{aligned}
& \dot{x}_{i}=1 / 4+x_{i}^{2}+\sum_{j=1}^{n}\left(c_{i j}+h_{i j} x_{i}\right) s_{j}+\mathcal{O}(\sqrt{\mu}), \\
& \dot{s}_{i}=\varepsilon\left\{x_{i}-p_{i} s_{i}+\mathcal{O}(\sqrt{\mu})\right\},
\end{aligned}
$$

where

$$
\begin{aligned}
c_{i j} & =\frac{1}{2} \frac{\partial g_{i}}{\partial y_{i}} \frac{\partial^{2} q_{i}}{\partial c_{j} \partial \mu}, \\
h_{i j} & =\frac{\partial g_{i}}{\partial y_{i}} \frac{\partial^{2} q_{i}}{\partial c_{j} \partial y_{i}}, \\
p_{i} & =-\frac{\partial g_{i}}{\partial c_{i}}
\end{aligned}
$$


and all derivatives are evaluated at the origin. Notice that each unperturbed $(\varepsilon=0)$ oscillator has a limit cycle attractor

$$
\gamma_{i}(t)=\frac{1}{2} \tan \frac{t}{2} \subset \mathbb{R} \cup\{\infty\}
$$

with period $T=2 \pi$. From the normalization condition (1.7),

$$
Q_{i}(t)^{\top}\left(1 / 4+\gamma_{i}(t)^{2}\right)=1 \quad \text { and } \quad P_{i}(t)^{\top}\left(1 / 4+\gamma_{i}(t)^{2}\right)=-\gamma_{i}(t),
$$

we can find directly the solutions to the adjoint problem (1.6),

$$
Q_{i}(t)=2(1+\cos t) \quad \text { and } \quad P_{i}(t)=-\sin t,
$$

and use them in Theorem 1.1 to find all parameters and functions. It is easy to see that

$$
\begin{aligned}
a_{i j} & =\frac{1}{2 \pi} \int_{0}^{2 \pi} 2(1+\cos t)\left(c_{i j}+\frac{h_{i j}}{2} \tan \frac{t}{2}\right) d t=2 c_{i j}, \\
b_{i j} & =\frac{1}{2 \pi} \int_{0}^{2 \pi}-\sin t\left(c_{i j}+\frac{h_{i j}}{2} \tan \frac{t}{2}\right) d t=-\frac{h_{i j}}{2}, \quad i \neq j, \\
b_{i i} & =-\frac{h_{i i}}{2}-p_{i} .
\end{aligned}
$$

Next,

$$
\int_{0}^{t+\chi} \frac{1}{2} \tan \frac{\bar{t}}{2} d \bar{t}=-\ln \left|\cos \frac{t+\chi}{2}\right|
$$

and

$$
\begin{aligned}
H_{i j}(\chi) & =\frac{1}{2 \pi} \int_{0}^{2 \pi} 2(1+\cos t)\left(c_{i j}+\frac{h_{i j}}{2} \tan \frac{t}{2}\right)\left(-\ln \left|\cos \frac{t+\chi}{2}\right|\right) d t \\
& =c_{i j}(2 \ln 2-\cos \chi)+\frac{h_{i j}}{2} \sin \chi \\
K_{i j}(\chi) & =\frac{1}{2 \pi} \int_{0}^{2 \pi}-\sin t\left(c_{i j}+\frac{h_{i j}}{2} \tan \frac{t}{2}\right)\left(-\ln \left|\cos \frac{t+\chi}{2}\right|\right) d t \\
& =-\frac{c_{i j}}{2} \sin \chi-\frac{h_{i j}}{4}(2 \ln 2+\cos \chi), \quad i \neq j, \\
K_{i i}(0) & =-\frac{h_{i i}}{4}(2 \ln 2+1)-p_{i} \ln 2 .
\end{aligned}
$$

A typical form of the connection function $H_{i j}(\chi)$ and $K_{i j}(\chi)$ is depicted in Figure 2.3.

2.4.1. Two identical oscillators. Let us consider synchronization dynamics of two identical slowly coupled oscillators of the form

$$
\begin{array}{ll}
\dot{x}_{1}=1 / 4+x_{1}^{2}+\left(c+h x_{1}\right) s_{2}, & \dot{x}_{2}=1 / 4+x_{2}^{2}+\left(c+h x_{2}\right) s_{1}, \\
\dot{s}_{1}=\varepsilon\left(x_{1}-p s_{1}\right), & \dot{s}_{2}=\varepsilon\left(x_{2}-p s_{2}\right)
\end{array}
$$

with $p>0$ and arbitrary $c$ and $h$. The canonical phase model has the form

$$
\begin{aligned}
& \dot{\varphi}_{1}=2 c u_{2}+H\left(\varphi_{2}-\varphi_{1}\right), \\
& \dot{u}_{1}=-p\left(u_{1}+\ln 2\right)-\frac{h}{2}\left(u_{1}+u_{2}\right)+K\left(\varphi_{2}-\varphi_{1}\right), \\
& \dot{\varphi}_{2}=2 c u_{1}+H\left(\varphi_{1}-\varphi_{2}\right), \\
& \dot{u}_{2}=-p\left(u_{2}+\ln 2\right)-\frac{h}{2}\left(u_{1}+u_{2}\right)+K\left(\varphi_{1}-\varphi_{2}\right),
\end{aligned}
$$




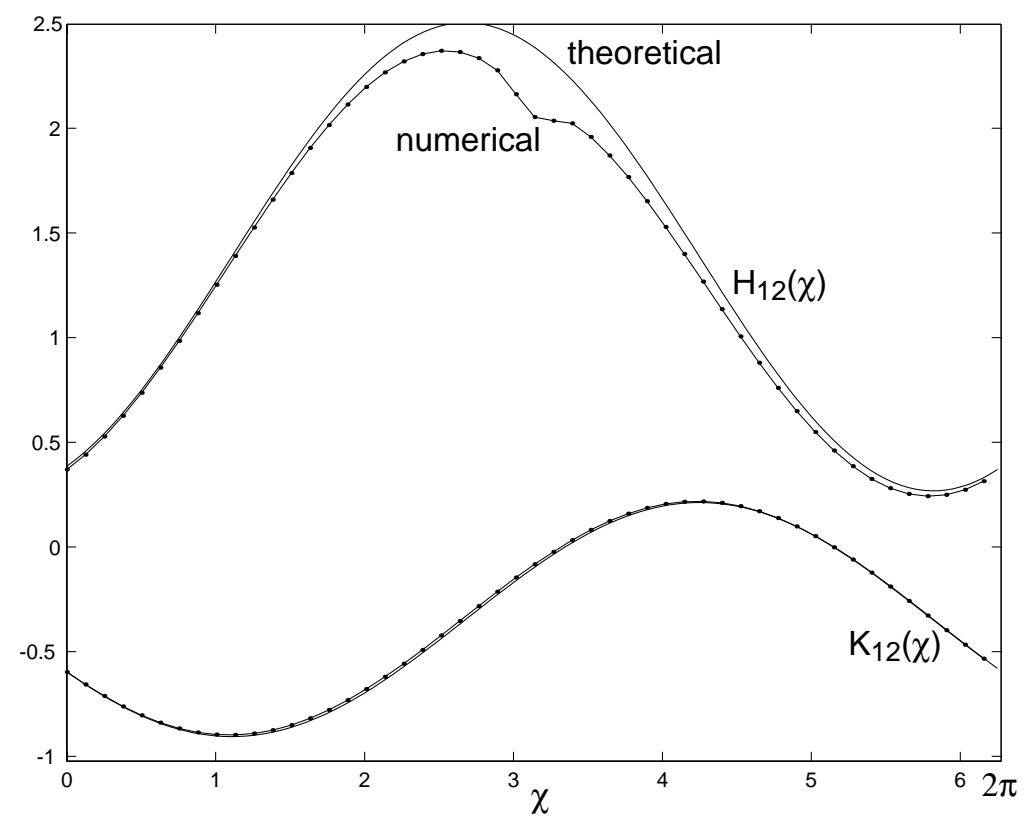

FIG. 2.3. Numerical (see Appendix B) and analytical forms of the connection functions for slowly coupled oscillators near a saddle-node on invariant circle bifurcation. Parameters in (2.6), (2.7): $c_{12}=h_{12}=p_{1}=1$.

where

$$
H(\chi)=c(2 \ln 2-\cos \chi)+\frac{h}{2} \sin \chi \quad \text { and } \quad K(\chi)=-\frac{c}{2} \sin \chi-\frac{h}{4}(2 \ln 2+\cos \chi) .
$$

Let $\chi=\varphi_{2}-\varphi_{1}$ and $u=u_{2}-u_{1}$; then

$$
\begin{aligned}
& \dot{\chi}=-2 c u-h \sin \chi \\
& \dot{u}=-p u+c \sin \chi
\end{aligned}
$$

The in-phase synchronized solution corresponds to the equilibrium $\chi=0$ and $u=0$ with the Jacobian matrix

$$
L=\left(\begin{array}{cc}
-h & -2 c \\
c & -p
\end{array}\right)
$$

It is stable when

$$
\operatorname{tr} L=-h-p<0 \quad \text { and } \quad \operatorname{det} L=h p+2 c^{2}>0,
$$

which is always the case when $h>0$. (It is an easy exercise to check that the antiphase solution $\chi=\pi, u=0$ is stable when $h<p$ and $h p+2 c^{2}<0$.)

We see that in contrast to weak coupling, which leads to a neutrally stable synchronized state of two Class 1 identical oscillators (Hansel, Mato, and Meunier (1995), Ermentrout (1996), Izhikevich (1999)), slow coupling with $h>0$ always results in stability of the synchronized state, as we illustrate in Figure 2.4, regardless of the sign of the connection coefficient $c$. Notice that the convergence to the in-phase synchronized state $\chi=0$ is oscillatory, as we illustrate in Figure 2.5(a); that is, the oscillators 


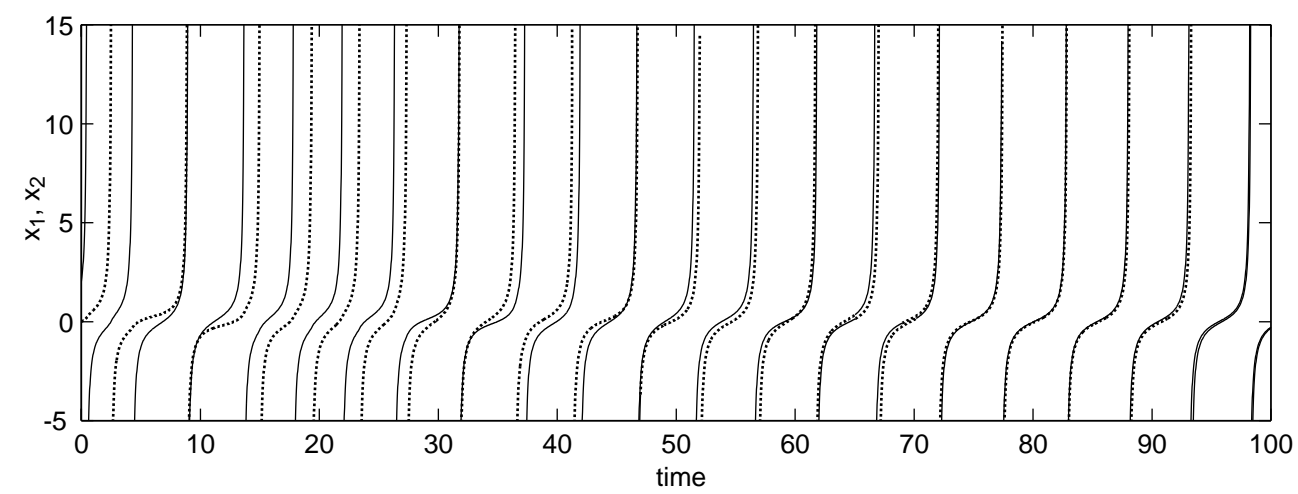

FIG. 2.4. Synchronization dynamics of two identical oscillators near a saddle-node on invariant circle bifurcation. Parameters: $c=5, h=p=1$, and $\varepsilon=0.05$.
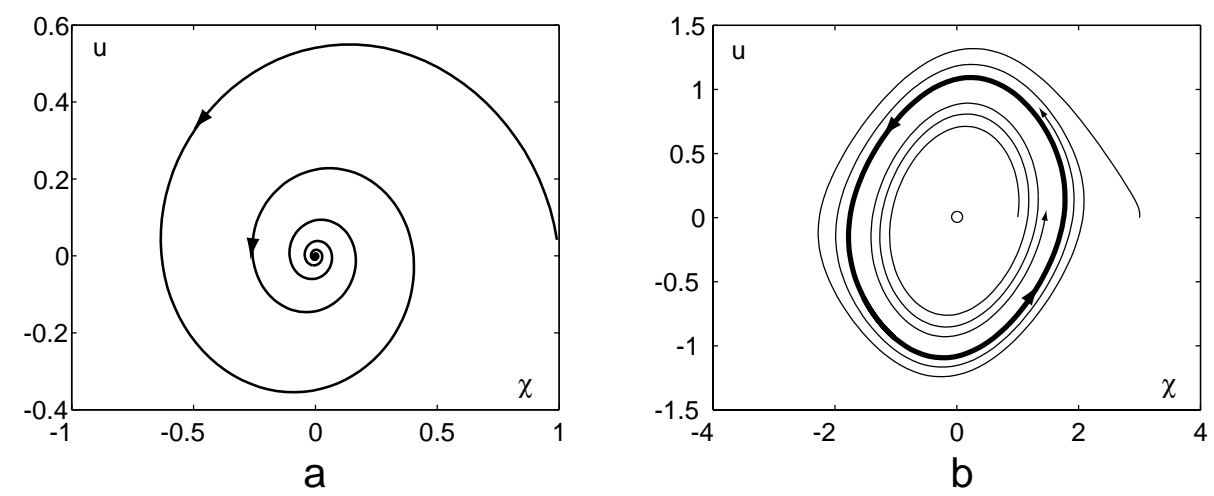

FIG. 2.5. (a) Convergence to the in-phase synchronized state is oscillatory (parameters as in Figure 2.4). (b) The phase difference $\chi$ may oscillate (here $h=-1.5$ ).

in Figure 2.4 take turns - a prominent feature of slowly connected networks that was discovered by Frankel and Kiemel (1993). If we decrease $h$ past $-p$, a small amplitude limit cycle may appear via a supercritical Andronov-Hopf bifurcation, and the phase difference $\chi$ would exhibit sustained oscillations, as shown in Figure 2.5(b).

2.5. Relaxation oscillators. Now we consider a slowly coupled network of relaxation oscillators of the form

$$
\begin{aligned}
\mu \dot{x}_{i} & =F_{i}\left(x_{i}, y_{i}\right)+q_{i}\left(x_{i}, y_{i}, s_{1}, \ldots, s_{n}\right), \\
\dot{y}_{i} & =G_{i}\left(x_{i}, y_{i}\right)+r_{i}\left(x_{i}, y_{i}, s_{1}, \ldots, s_{n}\right), \\
\dot{s}_{i} & =\varepsilon g_{i}\left(x_{i}, y_{i}, s_{i}\right),
\end{aligned}
$$

where $\mu \ll 1, x_{i}, y_{i}, s_{i} \in \mathbb{R}$, and

$$
q_{i}\left(x_{i}, y_{i}, 0, \ldots, 0\right)=0, \quad r_{i}\left(x_{i}, y_{i}, 0, \ldots, 0\right)=0, \quad \text { and } \quad g_{i}(0,0,0)=0 .
$$

Suppose that each unperturbed $(\varepsilon=0, s=0)$ system

$$
\begin{aligned}
\mu \dot{x}_{i} & =F_{i}\left(x_{i}, y_{i}\right), \\
\dot{y}_{i} & =G_{i}\left(x_{i}, y_{i}\right)
\end{aligned}
$$



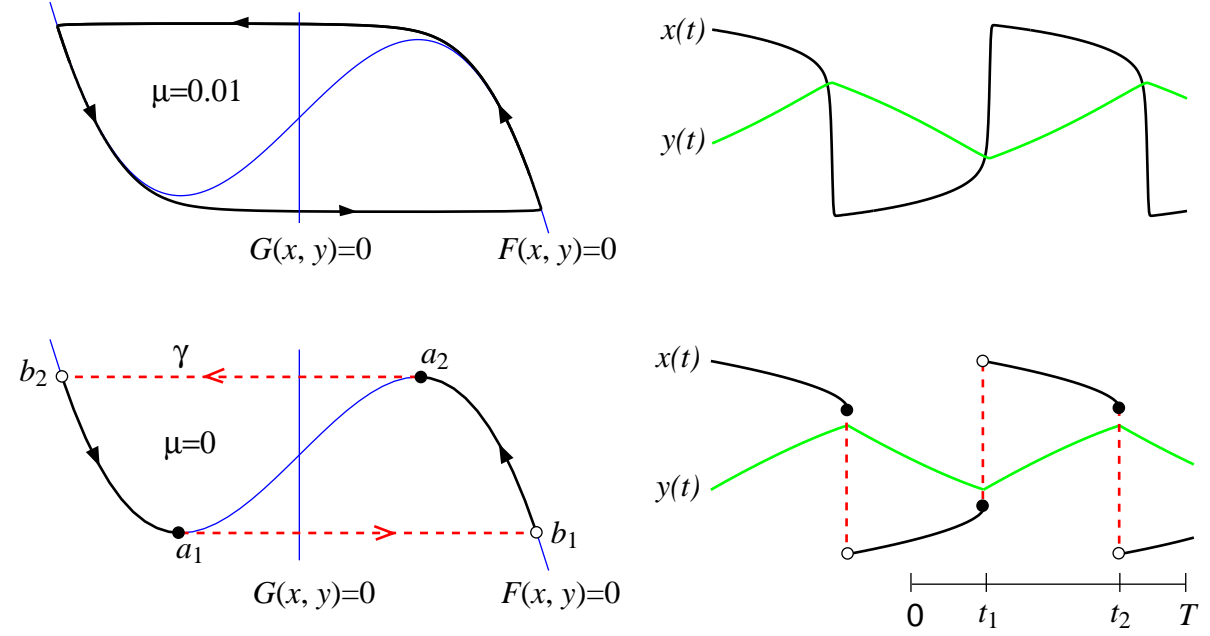

FIG. 2.6. Top: Nullclines and periodic solution of the van der Pol relaxation oscillator $\mu \dot{x}=$ $-y+x-x^{3} / 3, \dot{y}=x$ for $\mu=0.01$. Bottom: The periodic solution becomes discontinuous in the limit $\mu \rightarrow 0$. (Modified from Izhikevich (2000)).

has a relaxation limit cycle attractor with period $T>0$ converging to $\gamma_{i}(t) \subset \mathbb{R}^{2}$ similar to the one depicted in Figure 2.6 in the limit $\mu \rightarrow 0$. Such an oscillation $\gamma_{i}(t)$ has two discontinuities (jumps) at $t=t_{1}$ and $t=t_{2}$. Izhikevich (2000) has shown that in this case the solution to the adjoint problem (1.6) converges as $\mu \rightarrow 0$ to

$Q_{i}(t)=\frac{1}{G_{i}\left(\gamma_{i}(t)\right)}\left(-\frac{\partial G_{i}}{\partial x_{i}}\left(\gamma_{i}(t)\right)\left(\frac{\partial F_{i}}{\partial x_{i}}\left(\gamma_{i}(t)\right)\right)^{-1}, 1\right)^{\top} \quad$ when $t \neq t_{1}$ and $t \neq t_{2}$

and

$$
Q_{i}\left(t_{k}\right)=\left(c_{k} \delta\left(t-t_{k}\right), \frac{1}{G_{i}\left(\gamma_{i}\left(a_{k}\right)\right)}\right)^{\top}
$$

where

$$
c_{k}=\left(\frac{\partial F_{i}}{\partial y_{i}}\left(\gamma_{i}(t)\right)\right)^{-1}\left(\frac{1}{G_{i}\left(a_{k}\right)}-\frac{1}{G_{i}\left(b_{k}\right)}\right)
$$

and $a_{k}$ and $b_{k}$ are the end points of the $k$ th jump, $k=1$, 2; see Figure 2.6.

Knowing $Q_{i}(t)$ and $P_{i}(t)$, one can easily find $a_{i j}, b_{i j}, H_{i j}$, and $K_{i j}$. For example,

$$
\begin{aligned}
& H_{i j}(\chi)=-\frac{1}{T} \int_{0}^{T} \frac{\frac{\partial G_{i}}{\partial x_{i}}\left(\gamma_{i}(t)\right)\left(\frac{\partial F_{i}}{\partial x_{i}}\left(\gamma_{i}(t)\right)\right)^{-1} \frac{\partial q_{i}}{\partial s_{j}}\left(\gamma_{i}(t)\right)-\frac{\partial r_{i}}{\partial s_{j}}\left(\gamma_{i}(t)\right)}{G_{i}\left(\gamma_{i}(t)\right)} \int_{0}^{t+\chi} g_{j}\left(\gamma_{i}(\bar{t})\right) d \bar{t} d t \\
&(2.8) \quad+\frac{1}{T} \sum_{k=1}^{2} c_{k}^{\top} \frac{\partial q_{i}}{\partial s_{j}}\left(a_{k}\right) \int_{0}^{t_{k}+\chi} g_{j}\left(\gamma_{j}(\bar{t})\right) d \bar{t} .
\end{aligned}
$$

A salient feature of weakly coupled relaxation oscillators is the existence of discontinuities in the connection functions $H_{i j}(\chi)$, which result in many interesting synchronization properties, such as superconvergence, persistence under perturbations of natural frequencies, etc. (see the discussion in Izhikevich (2000)). However, the 


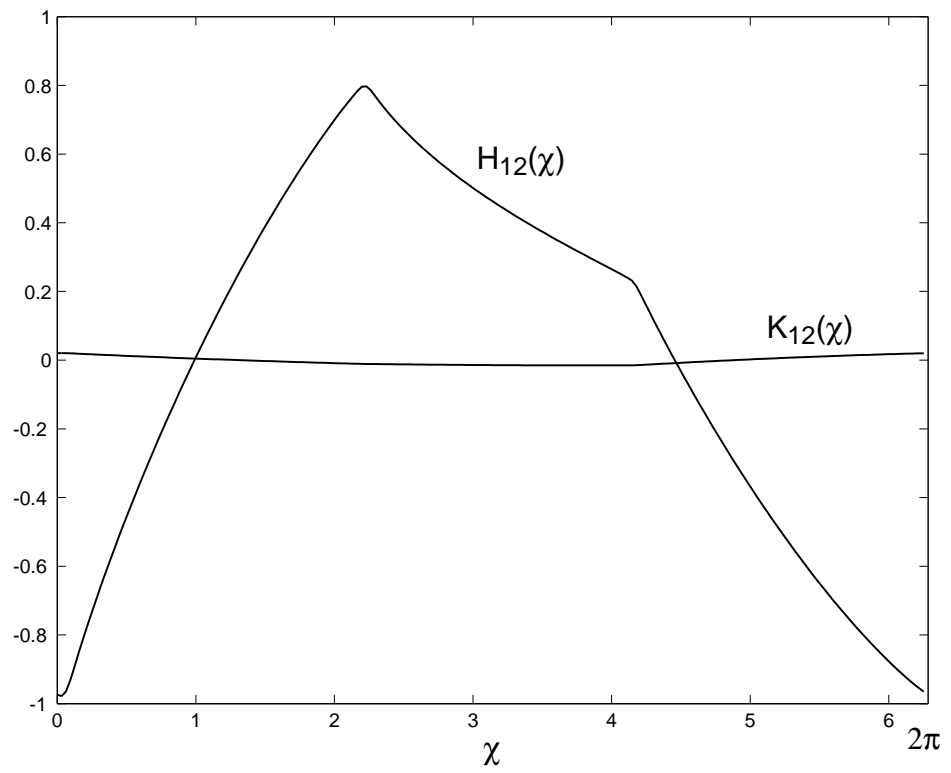

FIG. 2.7. Numerically found connection functions for slowly coupled Bonhoeffer-van der Pol relaxation oscillators $\mu \dot{x}_{i}=x_{i}-x_{i}^{3} / 3-y_{i}+s_{j}, \quad \dot{y}_{i}=0.5+x_{i}, \quad \dot{s}_{i}=\varepsilon\left(x_{i}-s_{i}\right), i=1,2, j=2,1$, $\mu=0.001$.

connection functions $H_{i j}(\chi)$ (and $K_{i j}(\chi)$ ) for slowly coupled relaxation oscillators are continuous, as shown in Figure 2.7. Hence, slowly coupled relaxation oscillators do not have those interesting synchronization properties.

3. Discussion. Much research in theoretical neuroscience is devoted to weakly coupled oscillators, as reviewed in Chapter 9 of Hoppensteadt and Izhikevich (1997). Slow connections, however, received less attention despite the fact that slow synaptic transmission is ubiquitous in the brain. Indeed, GABAb and NMDA receptor dynamics occur on the time scale of $150 \mathrm{~ms}$, which are much slower than the period of firing of many neurons, which is often smaller than $10 \mathrm{~ms}$. Purely NMDA synaptic connections have been found in the hippocampus (Isaac, Nicoll, and Malenka (1995)) and in the thalamocortical system (Isaac et al. (1997)). They are often referred to as being "silent synapses" since activation of NMDA receptors requires postsynaptic depolarization. (Most synapses have slow NMDA and fast AMPA glutamate receptors and hence describe slow and weak connections; see Remark 1.3.) Here we speculate that periodically firing neurons connected via NMDA or GABAb receptors could have quite different synchronization properties from the same neurons coupled via fast GABAa or AMPA receptors.

There have been only a few attempts to study rigorously slowly coupled oscillators. Notably, Rinzel and Frankel (1992) and Ermentrout (1994) used averaging to study dynamics of slowly coupled Hodgkin-Huxley-type models. They discovered new regimes that were not seen in weakly coupled networks, such as instabilities of relative phases and network bursting. Bressloff and Coombes (2000) applied similar methods to study integrate-and-fire neurons with slow synapses. The most important contribution was made by Frankel and Kiemel (1993) who showed how two slowly coupled oscillators can be transformed into a canonical phase model by an appropriate change of variables. 
In this paper we extend the results of Frankel and Kiemel (1993) to a network of $n \geq 2$ slowly coupled oscillators. We confirm that synchronization properties of such a network are described by a canonical phase model in which each oscillator is represented by a pair of variables $\varphi_{i}$ and $u_{i}$ on a cylindrical phase space. Using Malkin's theorem we can derive an analytical form for all coefficients and all connection functions $H_{i j}$ and $K_{i j}$ for the phase model.

In the second half of the paper we consider a few analytically solvable examples. First, we study oscillators near an Andronov-Hopf bifurcation and show that dynamics of variables $u_{i}$ do not depend on the phases $\varphi_{i}$. While the variables $u_{i}$ can slow down the convergence to a synchronized state, they cannot change the stability of that state.

Next, we study synchronization properties of Class 1 oscillators; that is, oscillators that are near a saddle-node on invariant circle bifurcation (Figure 2.2). Such a bifurcation results in periodic activity with arbitrarily small frequency, and it is believed to be involved in excitable properties of neocortical neurons in mammalian brains. It is well known (Hansel, Mato, and Meunier (1995), Ermentrout (1996)) that identical Class 1 oscillators if coupled weakly do not synchronize; more precisely, the synchronized state is neutrally stable for $n=2$ oscillators and unstable for $n>2$ oscillators (Izhikevich (1999)) regardless of whether the connections are excitatory or inhibitory. In contrast, slowly connected Class 1 oscillators do synchronize for both excitatory and inhibitory connections. We show this analytically using the canonical phase model approach, and then illustrate it numerically in Figure 2.4. Notice that the oscillators take turns, i.e., change order of firing, during the convergence to the synchronized state. This is a salient feature of slowly connected oscillators found by Frankel and Kiemel (1993) that cannot occur in weakly coupled networks of $n=2$ oscillators.

Finally, we consider slowly coupled relaxation oscillators and show that the connection functions $H_{i j}(\chi)$ and $K_{i j}(\chi)$ are continuous. Therefore, in contrast to weak coupling, slow coupling of relaxation oscillators does not lead to superstability of the in-phase synchronized solution (Izhikevich (2000)).

Appendix A. Malkin's theorem. Below we provide a general statement of Malkin's theorem (Malkin (1949), (1956)). A one-page proof can be found in (Hoppensteadt and Izhikevich (1997)).

Theorem A.1 (Malkin). Consider a T-periodic dynamical system of the form

$$
\dot{X}=F(X, t)+\varepsilon G(X, t, \varepsilon), \quad X \in \mathbb{R}^{m},
$$

and suppose that the unperturbed system, $\dot{X}=F(X, t)$, has a $k$-parameter family of T-periodic solutions

$$
X(t)=U(t, \alpha)
$$

where $\alpha=\left(\alpha_{1}, \ldots, \alpha_{k}\right)^{\top} \in \mathbb{R}^{k}$ is a vector of independent parameters, by which we mean that the rank of the $n \times k$ matrix $D_{\alpha} U$ is $k$. Suppose the adjoint linear problem

$$
\dot{R}_{i}=-\{D F(U(t, \alpha), t)\}^{\top} R_{i}
$$

has exactly $k$ independent $T$-periodic solutions $R_{1}(t, \alpha), \ldots, R_{k}(t, \alpha) \in \mathbb{R}^{m}$. Let $R$ be the matrix whose columns are these solutions such that

$$
R^{\top} D_{\alpha} U=I
$$


where $I$ is the identity $k \times k$ matrix. Then the perturbed system (A.1) has a solution of the form

$$
X(t)=U(t, \alpha(\varepsilon t))+\mathcal{O}(\varepsilon)
$$

where

$$
\frac{d \alpha}{d \tau}=\frac{1}{T} \int_{0}^{T} R(t, \alpha)^{\top} G(U(t, \alpha), t, 0) d t
$$

where $\tau=\varepsilon$ t is slow time. If (A.4) has a stable equilibrium, then system (A.1) has a T-periodic solution.

Appendix B. Numerical recipe. A good numerical method to solve the adjoint problem (1.6) was suggested by Williams and Bowtell (1997), and it is available in the Bard Ermentrout software package XPP. Here, for the sake of convenience, we present MATLAB script that uses the same method to determine all parameters and functions of Malkin's theorem.

The following MATLAB program consists of eight separate files, which are available at the first author's website. The user should provide the following parameters and functions:

- The period $\mathrm{T}$ and an initial point $\mathrm{x} 0$ on the limit cycle $\gamma(t)$ in the file main.m.

- The right-hand sides of the fast and slow systems in files $f . m$ and g.m, respectively.

- The parameters Np, NT, and ds in the file main.m, and $d x$ and dy in the file Df .m control the accuracy of the numerical method. They may be changed if necessary.

File main.m

function main

\% Eugene M. Izhikevich and Frank C. Hoppensteadt, December 19, 2001

$\%$ Determines all parameters and functions for two slowly

$\%$ coupled oscillators.

global s1 s2 Np T gamma

$\mathrm{Np}=200 ; \quad \%$ The number of points on the limit cycle

$\mathrm{NT}=200 ; \quad \%$ The number of iterations along the limit cycle

$\mathrm{T}=2 * \mathrm{pi} ; \quad \%$ The period of the limit cycle

$\mathrm{x} 0=[0.1 ; 0] ; \quad \%$ An initial point on the limit cycle

$\mathrm{s} 1=0 ; \mathrm{s} 2=0 ; \quad \%$ Steady-state value of $\mathrm{s}$

$[$ tg, gamma $]=\operatorname{ode} 23 \mathrm{~s}\left({ }^{\prime} f\right.$ ', $\left.(0: \mathrm{Np}-1) / \mathrm{Np} * \mathrm{~T}, \mathrm{x} 0\right)$;

figure(1),plot (tg, gamma); drawnow;

$\%$

$\%$ solve the adjoint for $Q(t)$ as $t->$-infty

[t,Qinv] = ode15s('adQ', $(0: \mathrm{NT} * \mathrm{~Np}-1) / \mathrm{Np} * \mathrm{~T},[1 ; 1])$;

$Q=Q \operatorname{inv}($ length $(\mathrm{t})-(0: \mathrm{Np}-1),:) ; \quad \% Q(\mathrm{t}) \Rightarrow \mathrm{Q}(-\mathrm{t})$

$Q=Q /(Q(1,:) * f(0$, gamma $(1,:)))$; $\quad$ Normalization

$\%$ solve the adjoint for $P(t)$ as $t \rightarrow-$ infty

$[t$, Pinv $]=\operatorname{ode} 15 \mathrm{~s}\left(\right.$ 'adP' $\left.^{\prime},(0: \mathrm{NT} * \mathrm{~Np}-1) / \mathrm{Np} * \mathrm{~T},[1 ; 1]\right)$;

$P=\operatorname{Pinv}($ length $(t)-(0: N p-1),:) ; \quad \% P(t) \Rightarrow P(-t)$

$\mathrm{P}=\mathrm{P}-\mathrm{Q} *(\mathrm{P}(1,:) * \mathrm{f}(0, \operatorname{gamma}(1,:))+\mathrm{g}(0, \operatorname{gamma}(1,:))) ; \%$ Normalization

figure (2), plot (tg, $Q, \operatorname{tg}, P)$; drawnow; 


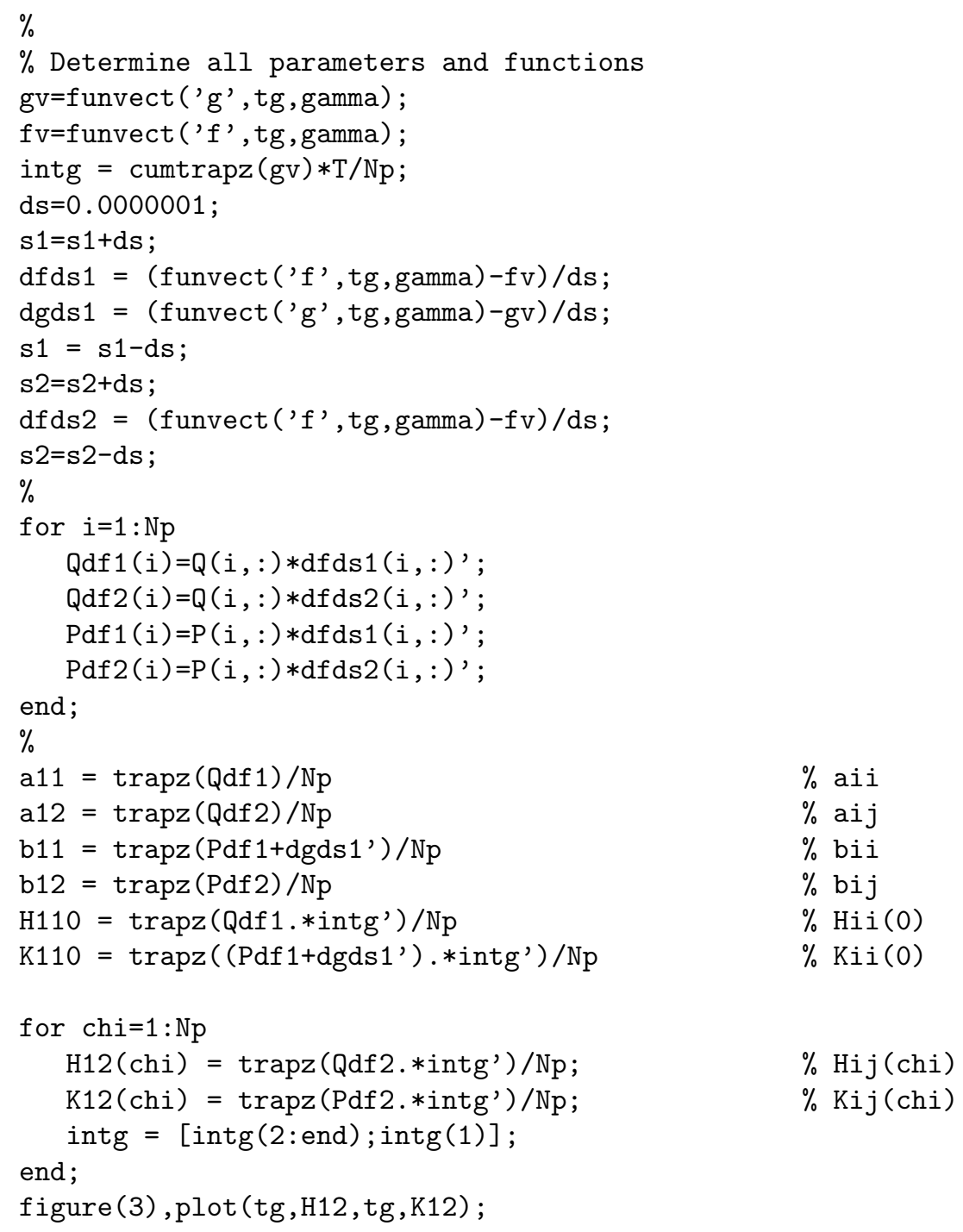

File $f . m$

function $x \operatorname{dot}=f(t, x)$

$\%$ Right-hand side of the fast system

File $g \cdot m$

function sdot $=g(t, x)$

$\%$ Right-hand side of the slow system

global s1

$\operatorname{sdot}=2 * x(1)-s 1$

File Df .m 
function $d=D f(t, x)$

$\%$ Numerical evaluation of Jacobian matrix Df at the point ( $t, x$ )

$\mathrm{dx}=0.0000001 ; \mathrm{dy}=0.0000001$;

$d=[(f(t, x+[d x ; 0])-f(t, x)) / d x(f(t, x+[0 ; d y])-f(t, x)) / d y] ;$

File Dg.m

function $d=\operatorname{Dg}(t, x)$

$\%$ Numerical evaluation of derivative $\mathrm{Dg}$ at $\mathrm{x}$

$\mathrm{dx}=0.0000001 ; \mathrm{dy}=0.0000001$;

$d=[(g(t, x+[d x ; 0])-g(t, x)) / d x(g(t, x+[0 ; d y])-g(t, x)) / d y] ;$

File adQ.m

function $Q \operatorname{dot}=\operatorname{adQ}(t, Q)$

$\%$ Right-hand side of the adjoint equation

$\%$ Integrating as $t->$-infty

global Np T gamma

Qdot $\left.=D f\left(t, \operatorname{gamma}(\operatorname{ceil}(\mathrm{Np} * \bmod (-\mathrm{t} / \mathrm{T}+0.5 / \mathrm{Np}, 1)),:)^{\prime}\right)\right)^{\prime} * \mathrm{Q}$;

File adP.m

function Pdot $=\operatorname{adP}(t, P)$

$\%$ Right-hand side of the adjoint equation for $\mathrm{P}$

$\%$ Integrating as $t \rightarrow-$ infty

global Np T gamma

gmt $\left.=\operatorname{gamma}(\operatorname{ceil}(N p * \bmod (-\mathrm{t} / \mathrm{T}+0.5 / \mathrm{Np}, 1)),)^{\prime}\right)^{\prime}$;

Pdot $=\operatorname{Df}(t, \text { gmt })^{\prime} * P+D g(t, g m t) '$;

File funvect.m

function ans $=$ funvect $($ fname, $t, x)$

$\%$ Applies function fname to the vector of arguments $t, x$

ans $=\operatorname{zeros}(\operatorname{length}(t), \operatorname{length}($ feval $($ fname, $t(1), x(1,:))))$;

for $i=1:$ length $(t)$

ans $(i,:)=$ feval (fname,t(i), $x(i,:))^{\prime}$;

end;

\section{REFERENCES}

P.C. Bressloff And S. Coombes (2000), Dynamics of strongly coupled spiking neurons, Neural Computation, 12, pp. 91-129.

G.B. Ermentrout (1994), Reduction of conductance-based models with slow synapses to neural nets, Neural Computation, 6, pp. 679-695.

G.B. ERmentrout (1996), Type I membranes, phase resetting curves, and synchrony, Neural Computation, 8, pp. 979-1001.

P. Frankel And T. KiEmel (1993), Relative phase behavior of two slowly coupled oscillators, SIAM J. Appl. Math., 53, pp. 1436-1446.

D. Hansel, G. Mato, and C. Meunier (1995), Synchrony in excitatory neural networks, Neural Computations, 7, pp. 307-335.

F.C. Hoppensteadt and E.M. Izhikevich (1997), Weakly Connected Neural Networks, SpringerVerlag, New York.

J.T. IsAaC, R.A. Nicoll, AND R.C. Malenka (1995), Evidence for silent synapses: Implications for the expression of LTP, Neuron, 15, pp. 427-34.

J.T. IsaAc, M.C. Crair, R.A. Nicoll, And R.C. Malenka (1997), Silent synapses during development of thalamocortical inputs, Neuron, 18, pp. 269-280. 
E.M. IzhikeVich (2000), Phase equations for relaxation oscillators, SIAM J. Appl. Math., 60, pp. 1789-1804.

E.M. IzHIKEVICH (1999), Class 1 neural excitability, conventional synapses, weakly connected networks, and mathematical foundations of pulse-coupled models, IEEE Trans. Neural Networks, 10, pp. $499-507$.

Y. Kuramoto (1984), Chemical Oscillations, Waves, and Turbulence, Springer-Verlag, New York.

I.G. Malkin (1949), Methods of Poincare and Liapunov in Theory of Non-linear Oscillations, Gostexizdat, Moscow (in Russian).

I.G. Malkin (1956), Some Problems in Nonlinear Oscillation Theory, Gostexizdat, Moscow (in Russian).

J. Rinzel and P. Frankel (1992), Activity patterns of a slow synapse network predicted by explicitly averaging spike dynamics, Neural Computation, 4, pp. 534-545.

T.L. Williams And G. Bowtell (1997), The calculation of frequency-shift functions for chains of coupled oscillators, with application to a network model of the lamprey locomotor pattern generator, J. Comput. Neurosci., 4, pp. 47-55. 